\title{
Circulating Monocytic Myeloid-Derived Suppressor Cells Are Elevated and Associated with Poor Prognosis in Acute Myeloid Leukemia
}

\author{
Huiping Wang, Qianshan Tao $\mathbb{D}$, Zhitao Wang, Qing Zhang, Hao Xiao, Mei Zhou, Yi Dong, \\ and Zhimin Zhai
}

Department of Hematology, The Second Hospital of Anhui Medical University, and Hematology Research Center, Anhui Medical University, Hefei, Anhui, China

Correspondence should be addressed to Zhimin Zhai; zzzm889@126.com

Received 4 August 2020; Revised 12 November 2020; Accepted 8 December 2020; Published 21 December 2020

Academic Editor: Viviana Vallacchi

Copyright (C) 2020 Huiping Wang et al. This is an open access article distributed under the Creative Commons Attribution License, which permits unrestricted use, distribution, and reproduction in any medium, provided the original work is properly cited.

Background. Monocytic myeloid-derived suppressor cells (M-MDSCs) characterized with the phenotype of CD14 ${ }^{+} \mathrm{HLA}_{-}$-DR ${ }^{\text {low/- }}$ have attracted a lot of attention in the field of human tumor immunology. However, little is known about the roles of M-MDSCs in acute myeloid leukemia (AML) as opposed to their multiple roles in solid tumors. Methods. We examined the frequencies of M-MDSCs identified for CD14 ${ }^{+} \mathrm{HLA}-\mathrm{DR} \mathrm{R}^{\text {low/- }}$ by flow cytometry in the peripheral circulating blood of 109 newly diagnosed adult patients with AML and 30 healthy controls (HC). Then, we, respectively, validated the clinic significance of circulating M-MDSCs on the relevance of spectral features for diagnostic stratification, induction therapy response, treatment effect maintenance, and long-term survival in AML. Results. Circulating M-MDSC frequencies of AML were significantly higher than those of $\mathrm{HC}$ both in $\mathrm{CD} 14^{+}$monocytes $(46.22 \% \pm 2.95 \%$ vs. $1.07 \% \pm 0.17 \%, p<0.01)$ and peripheral blood mononuclear cells (PBMCs) $(4.21 \% \pm 0.80 \%$ vs. $0.17 \% \pm 0.03 \%, p<0.01)$. Elevated circulating M-MDSCs in patients with AML were significantly associated with low complete remission (CR) rate, high relapse/refractory rate, and poor long-term survival, but had no correlation with common clinic risks and cytogenetic molecular risk categories. Conclusions. It was demonstrated that circulating M-MDSCs are elevated and associated with poor prognosis in AML, suggesting M-MDSCs might be a prognostic indicator for AML.

\section{Introduction}

Acute myeloid leukemia (AML) is a hematopoietic malignancy characterized by an accumulation of undifferentiated and functionally heterogeneous populations of leukemic blasts. Recently, a large number of evidences suggest that the proliferation, survival, and drug resistance of leukemic blasts are modulated by the tumor microenvironment (TME), which are composed of a variety of cells, extracellular matrix, and extracellular molecules. Especially, several individual immune cell subsets and immune molecules are also involved in the TME of AML [1]. CD14-positive monocytes with low HLA-DR or no HLA-DR expression $\left(\mathrm{CD} 14^{+}\right.$HLA$\mathrm{DR}^{\text {low/- }}$ monocytes) have been grouped into a larger class of immunosuppressive cells called monocytic myeloid-derived suppressor cells (M-MDSCs) [2-5]. M-MDSCs have attracted a lot of attention in the field of human tumor immunology, and their functional capacities related to the immunosuppressive mechanisms have been illustrated in recent years $[4,6]$. However, little is known about the roles of M-MDSCs in hematopoietic malignancy especially for AML as opposed to their multiple roles in solid tumors [7].

So far, only some studies have reported that the frequencies of M-MDSCs are higher in the peripheral blood of AML than that of healthy controls (HC) $[8,9]$. Those M-MDSCs may be derived from leukemic or apparently normal progenitors [8] and inhibit the activity of CD8 T cell [9]. But the clinical significance and related influencing factors of $\mathrm{M}$ MDSCs in AML is not clear. In the present study, we not only systematically detected the frequencies of M-MDSCs in the 
TABLE 1: Characteristics of 109 newly diagnosed patients with AML.

\begin{tabular}{|c|c|c|c|c|c|}
\hline Group & Subgroup & Number & Group & Subgroup & Number \\
\hline \multirow{2}{*}{ Gender } & Male & 62 & Cross lineage & Yes & 37 \\
\hline & Female & 47 & & No & 72 \\
\hline \multirow{2}{*}{ Age } & $<60$ y $(14-59)$ & 58 & Karyotype & Low risk & 28 \\
\hline & $\geq 60$ y $(60-91)$ & 51 & & Medium risk & 47 \\
\hline \multirow{8}{*}{ FAB classification } & M0 & 1 & & High risk & 15 \\
\hline & M1 & 4 & & No data & 19 \\
\hline & M2 & 33 & Fusion gene & RUNX1-RUNX1T1 & 9 \\
\hline & M3 & 19 & & $\mathrm{PML} / \mathrm{RAR} \alpha$ & 16 \\
\hline & M4 & 20 & & СВF $\beta-\mathrm{MYH} 11$ & 5 \\
\hline & M5 & 9 & & Others & 79 \\
\hline & M6 & 1 & Gene mutation & FLT3-ITD & 9 \\
\hline & $\mathrm{Mu}$ & 22 & & CEBPA & 6 \\
\hline \multirow{3}{*}{ WBC } & Low & 24 & & NMP1 & 5 \\
\hline & Middle & 26 & & C-kit & 3 \\
\hline & High & 59 & & Others & 86 \\
\hline \multirow{2}{*}{ AML type } & De novo & 93 & First induction & CR & 35 \\
\hline & Secondary & 16 & & Non-CR & 27 \\
\hline \multirow{2}{*}{ Extramedullar } & Yes & 13 & Curative effect & CCR & 27 \\
\hline & No & 96 & & $\mathrm{R} / \mathrm{R}$ & 35 \\
\hline
\end{tabular}

Note: AML: acute myeloid leukemia; FAB: Franch-American-Britain; Mu: unclassifiable AML in morphology; WBC: white blood cell; CR: complete remission; Non-CR: not complete remission; CCR: continued complete remission; R/R: relapse/refractory.

peripheral circulating blood of 109 newly diagnosed adult patients with AML but also evaluated the frequencies of circulating M-MDSCs and their correlation with various clinical parameters, short-term efficacy, and long-term survival in the pathogenesis of leukemia. Finally, we found that circulating M-MDSCs are significantly elevated and might predict undesirable clinic outcomes and poor long-term prognosis in AML.

\section{Methods}

2.1. Patients and Samples. This study included 109 newly diagnosed adult patients with AML and age-/sex-matched 30 healthy adult controls in the Second Hospital of Anhui Medical University between 2013 and 2019. All patients were characterized by their own diagnostic stratification and received standard induction chemotherapy followed by either consolidation therapy or allogeneic hematopoietic stem cell transplantation (allo-HSCT) in accordance with the Chinese guidelines for diagnosis and treatment of adult AML $[10,11]$. The detailed clinical characteristics of a total of 109 patients with AML are shown in Table 1. The samples of peripheral blood from all patients and controls were collected prior to any treatment and were evaluated within 6 hours. This study was approved by the Institutional Review Board Institutional of the Second Hospital of Anhui Medical University.

2.2. M-MDSCs Analysis. Peripheral blood mononuclear cells (PBMCs) were stratified on FicollHypaque (Amersham Biosciences, Sweden) and separated by centrifugation for
25 min (500 g). Subsequently, PBMCs were collected and washed with phosphate-buffered saline (PBS). After washing, $100 \mu \mathrm{L}$ PBMCs was incubated with monoclonal antibodies and analyzed by flow cytometry Cytomics ${ }^{\circledR}$ FC500 (Beckman Coulter, Miami, FL, USA), and EXPO 32 Multicomp software was used for data acquisition and analysis. The following monoclonal antibodies were purchased from Beckman Coulter (Miami, FL, USA): FITC-labeled CD14 (clone RMO52), PE-labeled HLA-DR (clone Immu-357), and PC5-labeled CD45 (clone J.33). M-MDSCs subsets were stained and identified by the phenotype of CD $14^{+} \mathrm{HLA}-\mathrm{DR}{ }^{\text {low/- }}$ in $\mathrm{CD} 45^{++}$cells. Isotype-matched controls were included in the experiments and were used to define the cutoff point for positive/negative staining.

2.3. Statistical Analysis. All statistical analysis was performed with SPSS 16.0 (IBM, Chicago, IL, USA). The Student's $t$ test, chi-squared test, and Fisher's exact probability tests were used as appropriate to evaluate the significance of the differences in data between different groups. If variances within groups were not homogeneous, a nonparametric MannWhitney test was used. Overall survival (OS) was used and defined as the time from date of diagnosis until the date of death. The prognostic value was evaluated by Kaplan-Meier survival curves. We used healthy adult controls as an indicator to generate the ROC curve and determined the optimal cutoff value based on the Yoden index. The cutoff values of M-MDSCs were $0.5 \%$. More than or equal to the respective cutoff value was defined as the high-frequency group, and less than the respective cutoff value was defined as the low- 


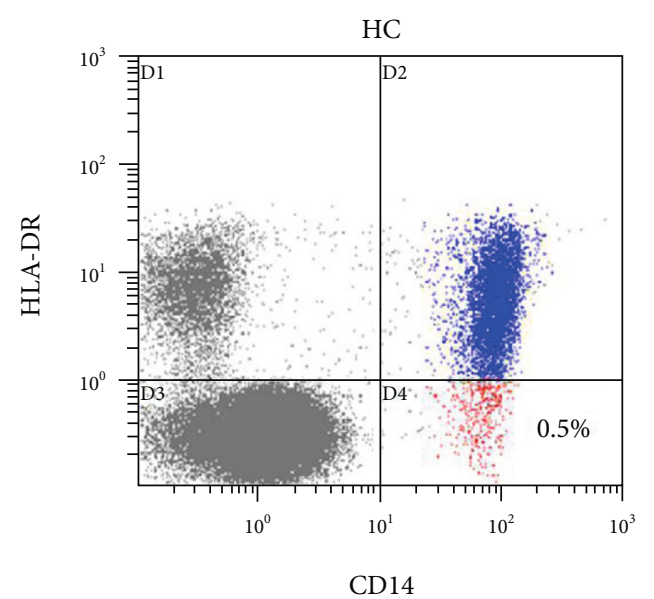

(a)

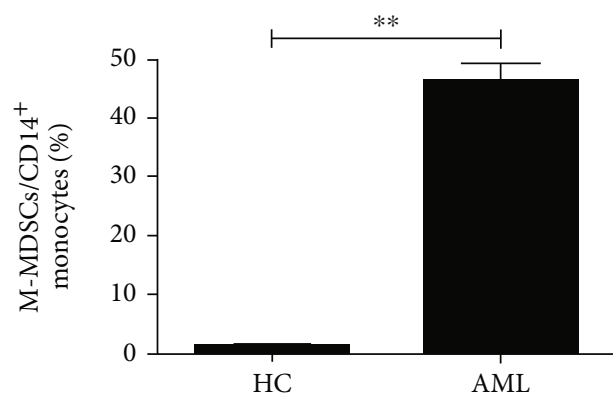

(c)

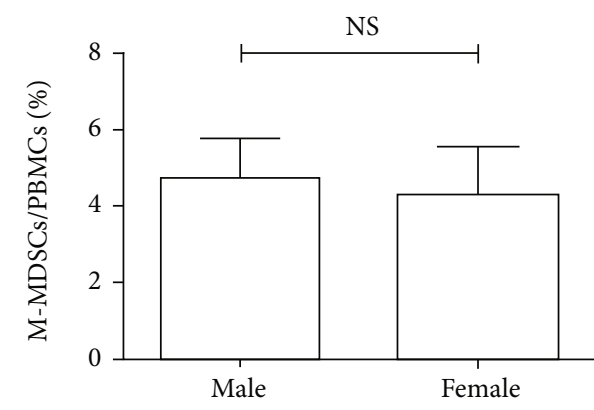

(e)

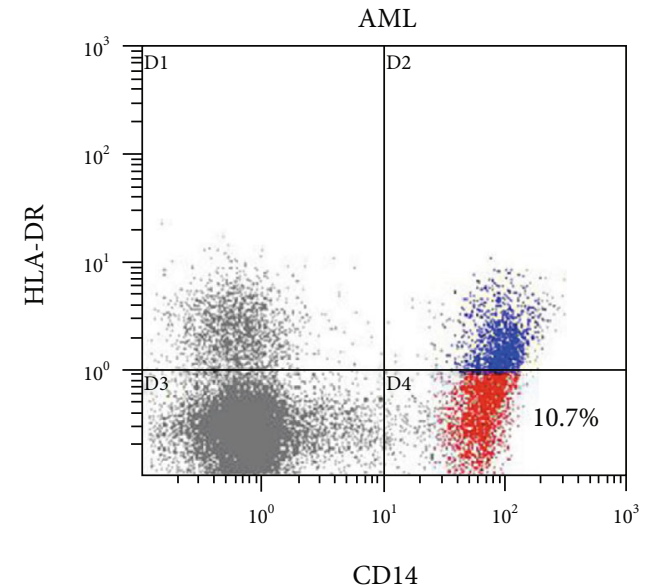

(b)

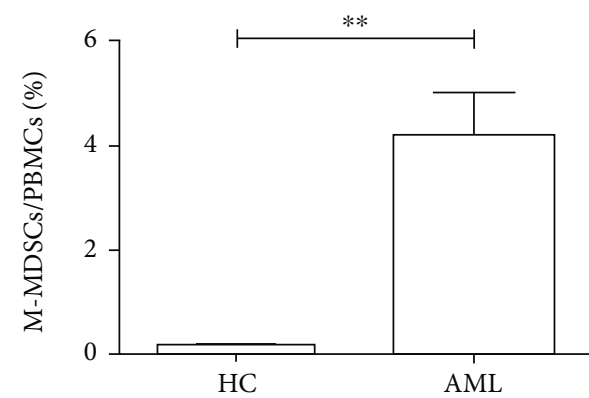

(d)

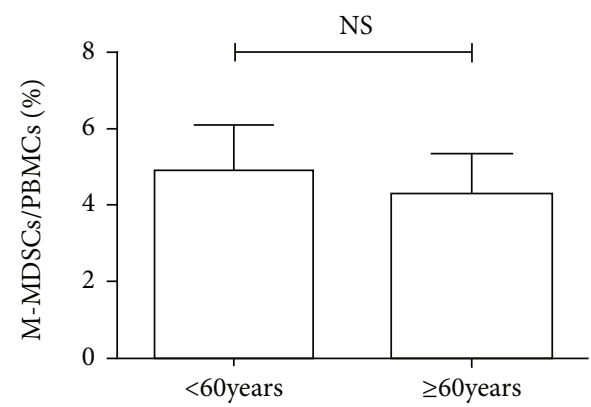

(f)

FIgURE 1: The frequencies of circulating M-MDSCs were elevated in AML. Representative flow cytometry dot plots demonstrated the frequencies of CD14 ${ }^{+}$HLA-DR ${ }^{\text {LOW/- }}$ M-MDSCs in AML (a) and HC (b). The frequencies of circulating M-MDSCs of AML ( $\left.n=109\right)$ and $\mathrm{HC}(n=30)$ in the percentage of $\mathrm{CD} 14^{+}$monocytes (c) and PBMCs (d). The frequencies of circulating M-MDSCs of AML in different gender groups (e) and age groups (f). AML: acute myeloid leukemia; HC: healthy controls; NS: not significant; ${ }^{* *} p<0.01$.

frequency group. The $p$ value less than 0.05 was considered statistically significant.

\section{Results}

3.1. Circulating M-MDSCs Were Elevated in AML. The frequencies of M-MDSCs in 109 newly diagnosed adult patients with $\mathrm{AML}$ and $30 \mathrm{HC}$ were investigated by flow cytometry in the present study. We found that circulating M-MDSCs frequencies of AML were significantly higher than those of $\mathrm{HC}$ both in $\mathrm{CD} 14^{+}$monocytes $(46.22 \% \pm 2.95 \%$ vs. $1.07 \%$ $\pm 0.17 \%, p<0.01$; Figures $1(\mathrm{a})$ and $1(\mathrm{c}))$ and PBMCs $(4.21 \% \pm 0.80 \%$ vs. $0.17 \% \pm 0.03 \%, p<0.01$; Figures 1 (b) and $1(\mathrm{~d})$ ). Thus, we alternatively used the frequencies of MMDSCs in PBMCs for further analysis in the following text. However, no significant differences in the circulating $\mathrm{M}$ MDSCs frequencies were found in the different gender and age groups (Figures 1(e) and 1(f)). Together, it was suggested that circulating M-MDSCs were elevated in AML, which was not affected by age and gender.

3.2. Circulating M-MDSCs Were Associated with Poor Prognosis in AML. 62 cases (57\%) of 109 patients with AML received standard treatment consisting of one or two cycles of induction chemotherapy followed by either consolidation therapy or allo-HSCT; the other 47 cases (43\%) only 


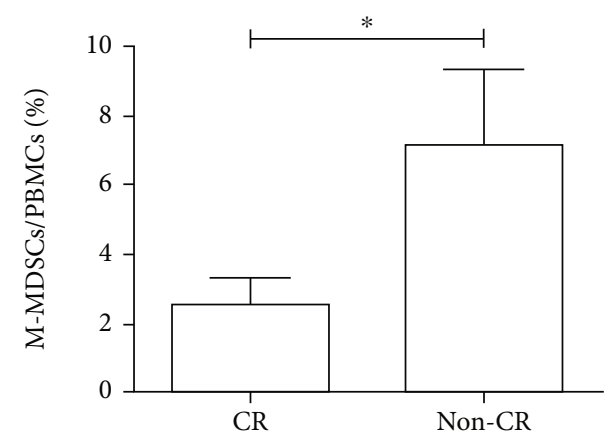

(a)

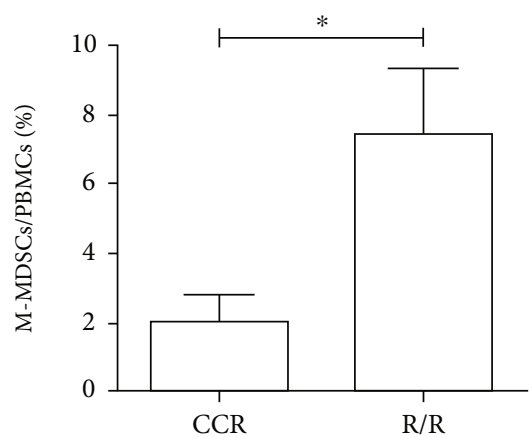

(b)

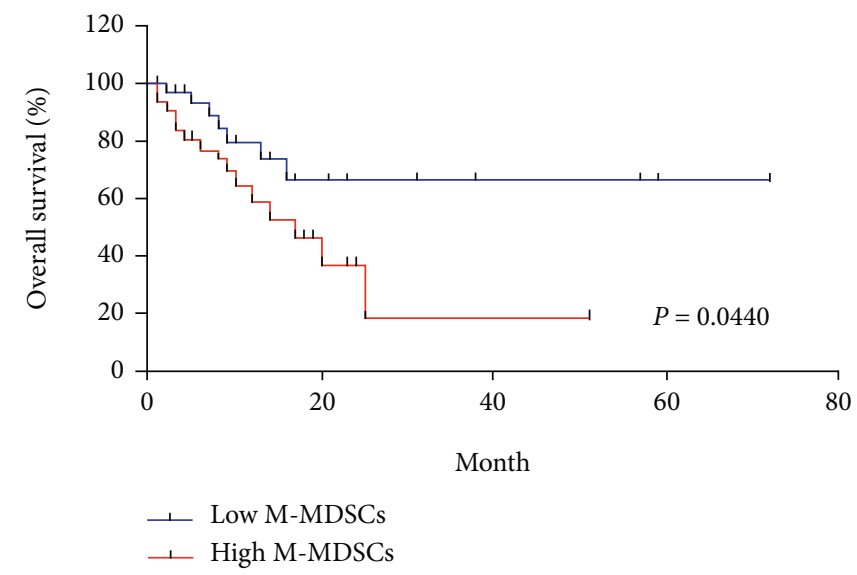

(c)

FIgURE 2: The prognostic relevance of circulating M-MDSCs in AML. The frequencies of circulating M-MDSCs in the CR group were significantly lower than the non-CR group (a). The frequencies of circulating M-MDSCs in the CCR group were significantly lower than the $\mathrm{R} / \mathrm{R}$ group (b). The low circulating M-MDSC frequency group had a significant survival advantage compared with the high group (c). ${ }^{*} p<0.05$.

received supportive care because of their old age or poor physical condition. In Table 1 and Figure 2(a), 35 of 62 cases (56\%) obtained complete remission (CR) and 27 of 62 cases (44\%) did not obtain CR (non-CR) after the first induction chemotherapy. The frequencies of circulating M-MDSCs in the CR group $(2.54 \% \pm 0.75 \%)$ were significantly lower than the non-CR group $(7.08 \% \pm 2.29 \%, p<0.05)$. In Table 1 and Figure 2(b), 27 of 62 cases (44\%) preserved continued complete remission (CCR) and 35 of 62 cases (56\%) eventually became refractory or relapsed $(\mathrm{R} / \mathrm{R})$ disease after the reinduction therapy or consolidation therapy/allo-HSCT. The frequencies of circulating M-MDSCs in the CCR group $(2.03 \% \pm 0.76 \%)$ were significantly lower than the $\mathrm{R} / \mathrm{R}$ group $(7.47 \% \pm 1.77 \%, p<0.05)$. For follow-up analyses, a total of 62 cases that received standard treatment were included. As shown in Figure 2(c), patients with the low circulating MMDSCs frequency had a significant survival advantage than patients with the high circulating M-MDSCs frequency $(p=0.0440)$. Together, it was suggested that elevated circulating M-MDSCs at the time of new diagnosis were a prognostic indicator to predict poor outcomes in patients with AML.

3.3. Circulating M-MDSCs Were Not Correlated with Common Clinic Risks in AML. In this article, we analyzed the correlation between the frequency of circulating $M$ -
MDSCs and common clinical risks in all 109 AML cases. In Figure 3(a), our data demonstrated that the frequencies of circulating M-MDSCs in French-American-British (FAB) classification system acute monocytic leukemia (M4)/acute myelomonocytic leukemia (M5) subtype groups were significantly higher than those in other FAB subtype groups $(10.74 \% \pm 2.29 \%$ vs. $2.29 \% \pm 0.53 \%, p<0.01)$. However, in Figures 3(b)-3(f), there were no significant statistical differences in circulating M-MDSCs in the other groups of AML type including de novo AML and secondary AML, white blood cell (WBC) count, cross linage expression, extramedullary infiltration, and leukemic blasts percentage (all $p>0.05$ ). Altogether, these data showed no correlation between circulating $M$ MDSCs and common clinic risks except for the FAB subtype.

3.4. Circulating M-MDSCs Were Not Correlated with Cytogenetic Molecular Risk Categories in AML. In the present study, 109 newly diagnosed patients with AML were enrolled. In Table 1, 90 cases (83\%) were detected of G-banded chromosome recognition and divided into the different groups of low risk, medium risk, and high risk, according to the Chinese guidelines for diagnosis and treatment of adult AML. 92 cases $(84 \%)$ were detected of 44 kinds of fusion genes. 40 cases (37\%) were detected of FLT3-ITD, NPM1, CEBPA, and Ckit gene mutations. Then, we analyzed the correlation between 


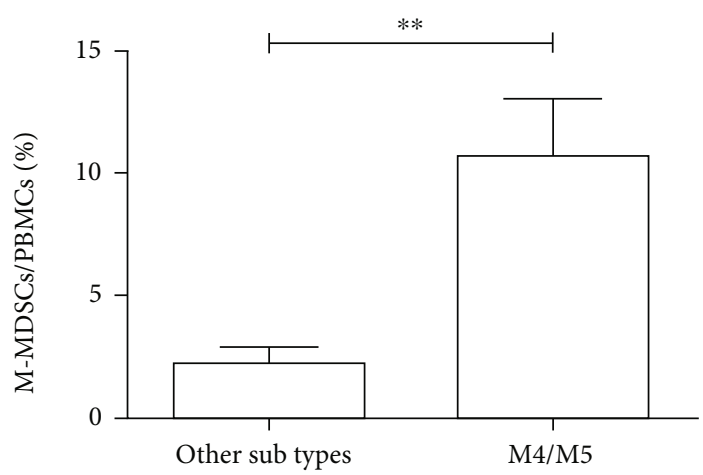

(a)

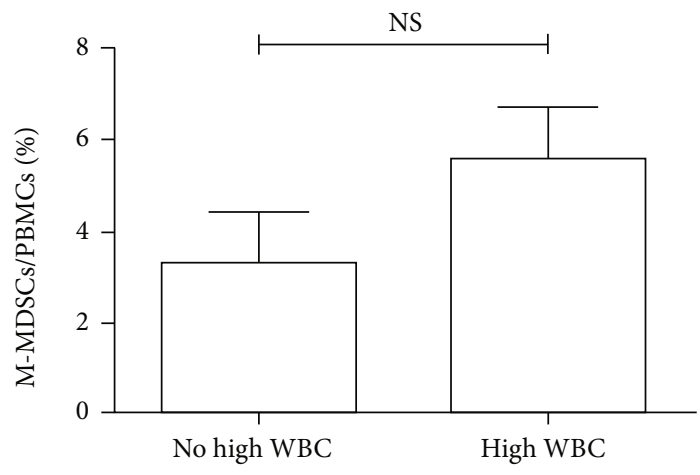

(c)

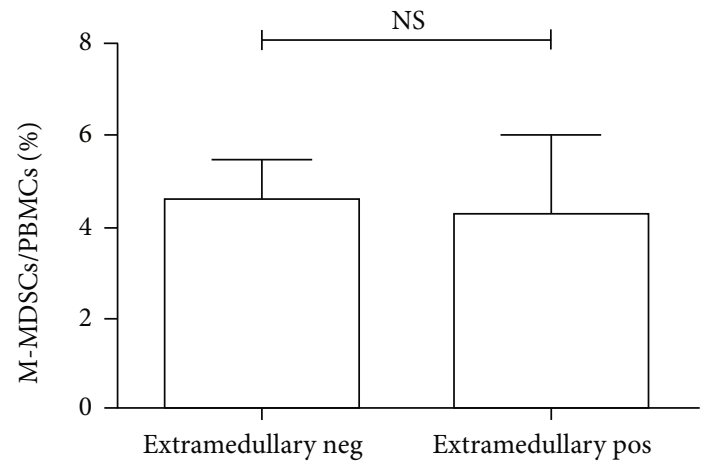

(e)

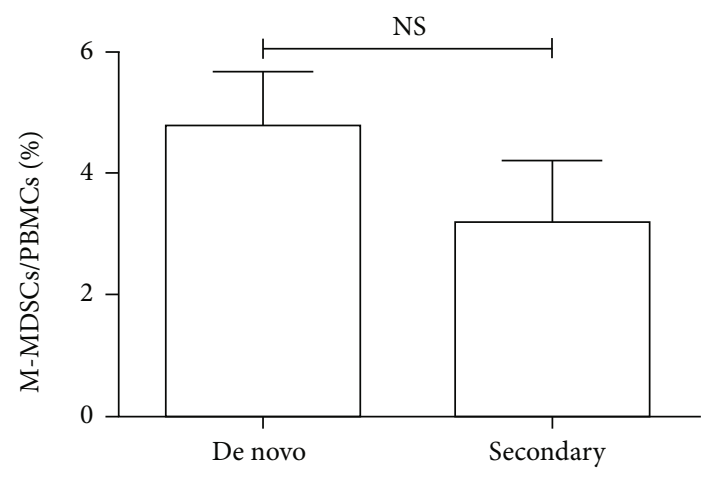

(b)

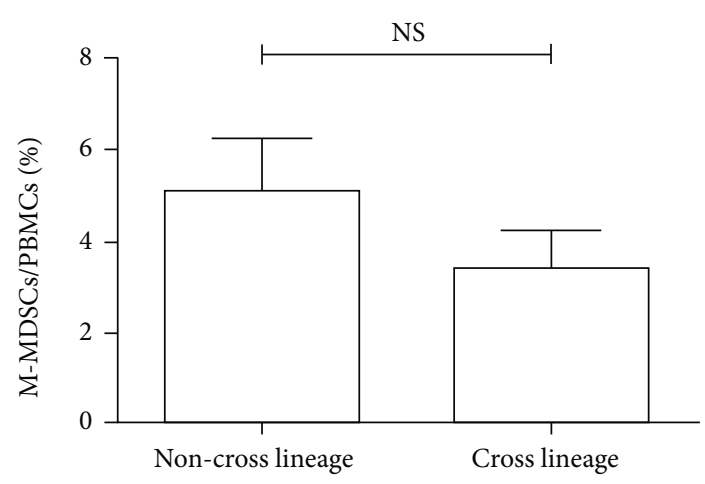

(d)

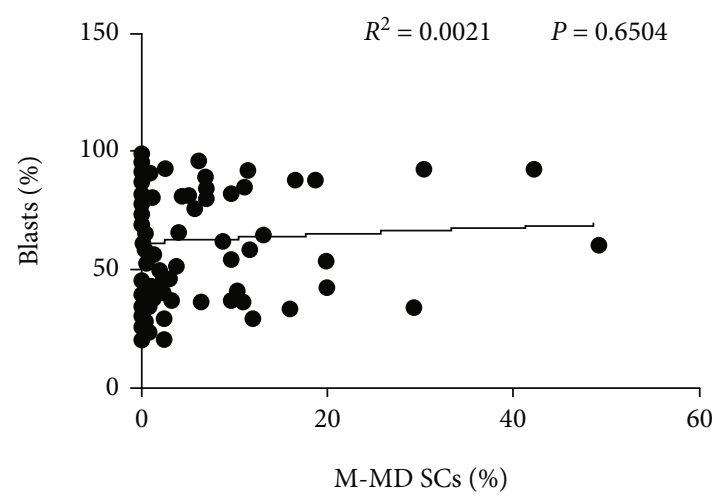

(f)

FIGURE 3: The correlation of circulating M-MDSC frequency and clinic risk categories in AML. The frequencies of circulating M-MDSCs in FAB M4/M5 subtype groups were significantly higher than the other FAB subtype groups (a). No significant statistical differences on circulating M-MDSCs were found in the different groups of AML type, WBC count, cross linage expression, extramedullary infiltration, and leukemic blasts percentage (b-f). NS: not significant; ${ }^{* *} p<0.01$.

the frequency of circulating M-MDSCs and karyotype stratification, fusion gene, and gene mutation. We found there were no significant statistical differences on circulating M-MDSCs in the groups of karyotype stratification, fusion gene, and gene mutation (Figures $4(\mathrm{a})-4(\mathrm{~h})$, all $p>0.05)$. Altogether, these data showed no correlation between circulating M-MDSCs and common cytogenetic molecular risk categories.

\section{Discussion}

In the late $1990 \mathrm{~s}, \mathrm{Gr} 1^{+} \mathrm{CD} 11 \mathrm{~b}^{+}$cells were suggested as defining the immunesuppressive myeloid cells which were func- tionally distinct from monocytes and neutrophils in mice $[12,13]$. The unique name "myeloid-derived suppressor cells (MDSCs)" of these immunesuppressive myeloid cells was proposed in 2007 [2]. In humans, monocytes that have diminished or not HLA-DR expression have emerged as important mediators of tumor-induced immunosuppression [14]. So, CD $14^{+}$HLA-DR ${ }^{\text {low/- }}$ monocytes have been grouped into a larger class of MDSCs and are commonly referred to as a special subtype of monocytic myeloid-derived suppressor cells (M-MDSCs) [14-16].

In recent years, great progress has been made in understanding the roles of M-MDSCs in human malignant tumors. 


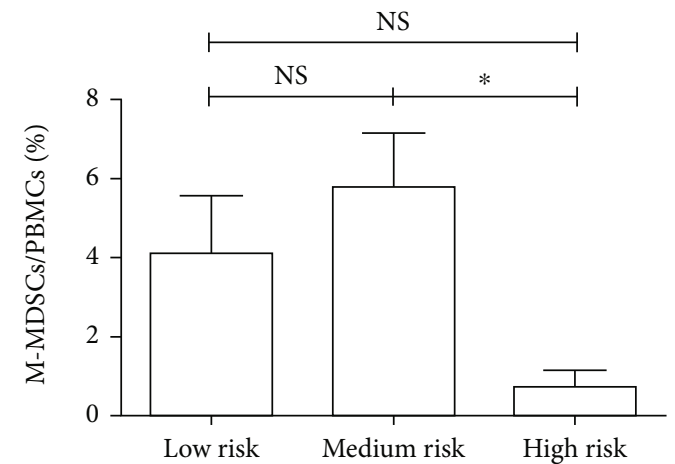

(a)

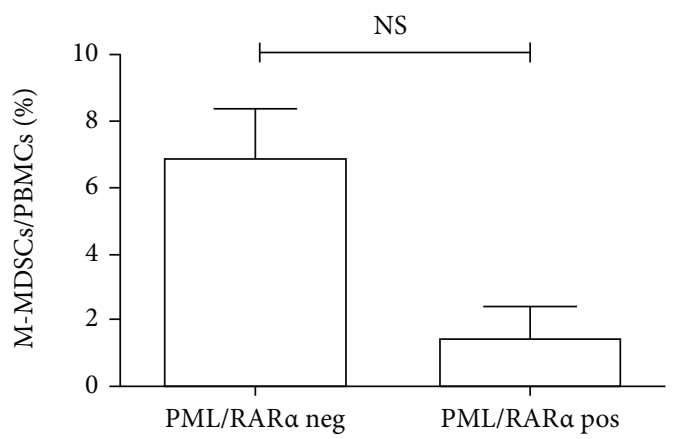

(c)

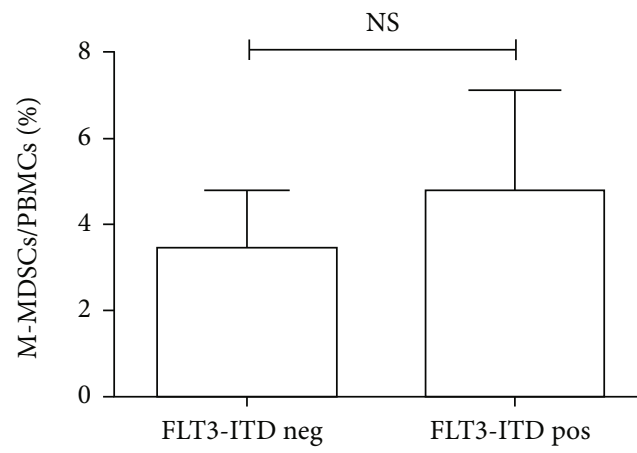

(e)

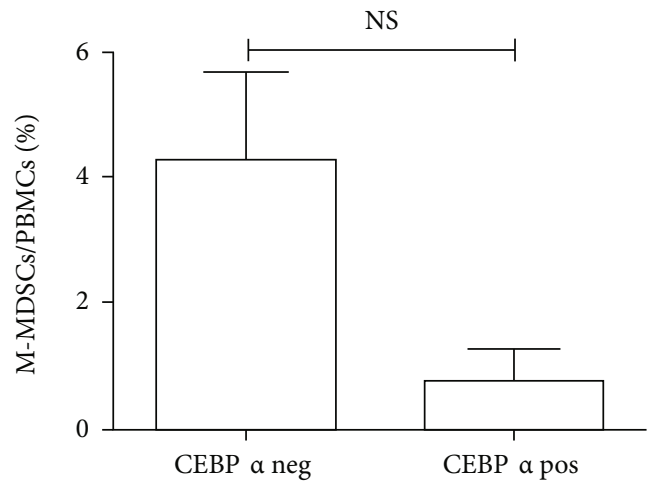

(g)

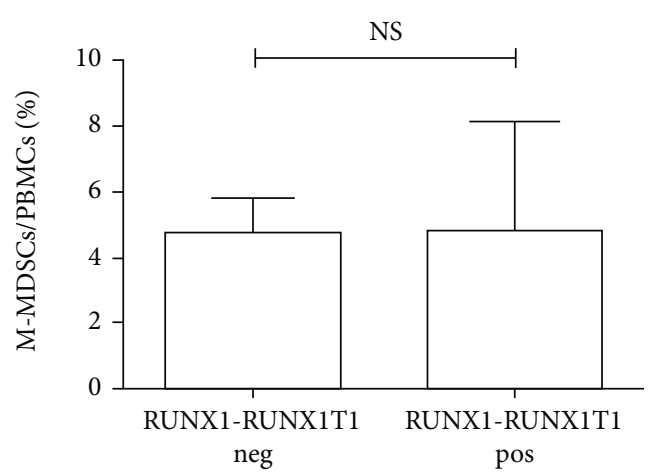

(b)

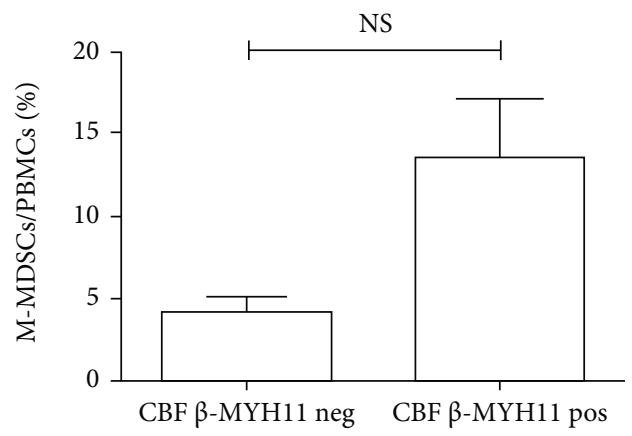

(d)

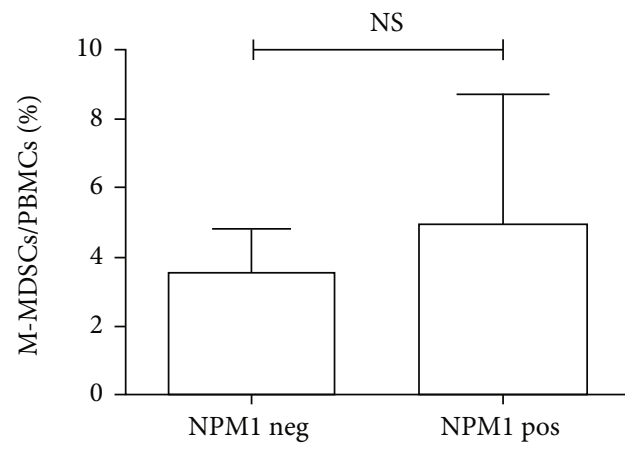

(f)

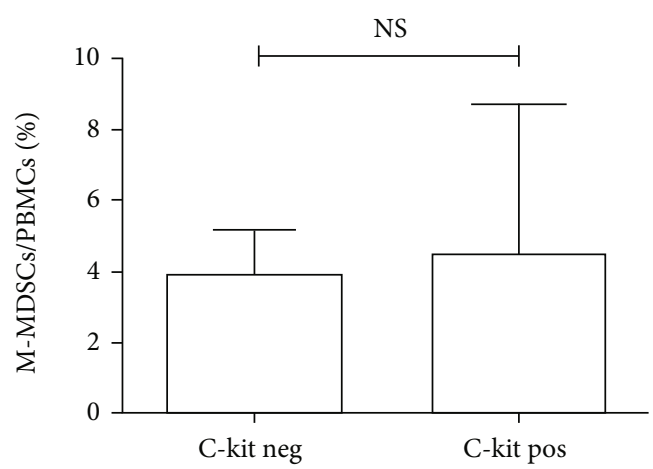

(h)

FIgURE 4: The correlation of circulating M-MDSC frequency and cytogenetic/molecular risk categories in AML. No significant statistical differences in circulating M-MDSCs were found in the different groups of karyotype stratification, fusion gene, and gene mutation (a-h). NS: not significant. 
Studies show that M-MDSCs are closely related to the tumor progression and treatment effect of patients with solid tumors $[17,18]$. For example, it has been reported that the frequencies of M-MDSCs are significantly increased in small-cell lung cancer, head and neck tumor, and bladder cancer, and closely related to the clinical stage of the tumor [19-21]. In patients with hematological malignancies, the researches on M-MDSCs are mostly focused on lymphoma and myeloma [7, 22]. In our previous studies, the result has shown that M-MDSCs are elevated and correlated with tumor progression, and even as an indicator for the efficacy of therapy in patients with myeloma. Meanwhile, we have reported that the plasma cells are able to induce the accumulation of M-MDSCs in vitro [23]. However, studies on M-MDSCs have been relatively limited in leukemia especially AML, not alike in solid tumors and lymphoma as well as myeloma.

AML is a heterogeneous hematopoietic malignancy that derives from aberrant proliferation and accumulation of leukemic blasts. Immunological disorders of several individual immune cell subsets and immune molecules have been shown to be related to the pathogenesis of AML [1]. In our previous studies, we have reported that tumor immune escape mechanism mediated by $\mathrm{CD} 4^{+} \mathrm{CD} 25^{+}$regulatory $\mathrm{T}$ cells (Tregs) and inhibitory cytokine IL-35 is a key factor for the proliferation and apoptosis of leukemic blasts [24, 25].

In this study, we further determined the proportion of MMDSCs by the analysis of flow cytometry in the peripheral circulating blood of 109 newly diagnosed adult patients with AML and 30 matched HC. Compared with the HC group, we found that circulating M-MDSCs frequencies both in CD14 monocytes and PBMCs were significantly increased in the AML group. Additionally, no significant difference in the proportion of circulating M-MDSCs was found in the different age and gender groups. So, it was illustrated that circulating M-MDSCs were elevated in AML, which might complicate with a severe immune dysfunction and contribute to patients' overall immune suppression.

Regarding the clinical values of M-MDSCs, our study showed that circulating M-MDSCs frequencies were significantly higher in the poor outcome patients with AML after the first induction of chemotherapy. The patients with high circulating M-MDSCs frequencies had a significantly shorter survival within 72 months of follow-up. These data were consistent with the same results of the study in solid tumor and lymphoma as well as myeloma $[18,26,27]$. It was suggested that elevated circulating M-MDSCs might be regarded as a prognostic indicator to predict the poor outcome in patients with AML. Moreover, we also analyzed the correlation between the frequency of M-MDSCs and common clinical and laboratory risk factors in patients with AML. We found that the high circulating M-MDSCs were only corrected to FAB M4/M5 subtypes mightily due to the increased proportion of monocytes in those special subtype patients. However, no other statistical correlation was found in AML types, WBC count, cross linage expression, extramedullary infiltration, leukemic blasts percentage, karyotype stratification, fusion gene, and gene mutation. Therefore, it was implied that M-MDSCs might be an independent prognostic indicator in AML.
In summary, circulating M-MDSCs were significantly elevated and closely related to the poor outcome of induction therapy in patients with AML. Circulating M-MDSCs could be used as a prognostic indicator to evaluate the survival of patients with AML. Targeting therapies for M-MDSCs might help to improve the host's antitumor immune function, which might be expected to become a new strategy for AML treatment in the future.

\section{Data Availability}

The data used to support the findings of this study are available from the corresponding author upon request.

\section{Ethical Approval}

This study was approved by the Institutional Review Board Institutional of the Second Hospital of Anhui Medical University.

\section{Conflicts of Interest}

The authors declare no conflict of interest.

\section{Authors' Contributions}

Zhimin Zhai designed the experiment. Huiping Wang, Qianshan Tao, and Hao Xiao performed all the experiments. Qianshan Tao wrote the manuscript. Qianshan Tao and Huiping Wang analyzed the data. Qianshan Tao, Qing Zhang, and Yi Dong collected the clinical specimens. Huiping Wang, Qianshan Tao, Qing Zhang, and Mei Zhou contributed to clinical data acquisition. All authors have read and agreed to the published version of the manuscript. Huiping Wang and Qianshan Tao contributed equally to this work.

\section{Acknowledgments}

This work was supported by grants from the National Natural Science Foundation of China (Number: 81670179) and the University Natural Science Research Project of Anhui Province (Number: KJ2019A0254).

\section{References}

[1] A. Isidori, V. Salvestrini, M. Ciciarello et al., "The role of the immunosuppressive microenvironment in acute myeloid leukemia development and treatment," Expert Review of Hematology, vol. 7, no. 6, pp. 807-818, 2014.

[2] D. I. Gabrilovich, V. Bronte, S. H. Chen et al., "The terminology issue for myeloid-derived suppressor cells," Cancer Research, vol. 67, no. 1, p. 425, 2007.

[3] D. I. Gabrilovich, S. Ostrand-Rosenberg, and V. Bronte, "Coordinated regulation of myeloid cells by tumours," Nature Reviews Immunology, vol. 12, no. 4, pp. 253-268, 2012.

[4] D. I. Gabrilovich, "Myeloid-derived suppressor cells," Cancer Immunology Research, vol. 5, no. 1, pp. 3-8, 2017.

[5] A. E. Mengos, D. A. Gastineau, and M. P. Gustafson, "The CD14+HLA-DRlo/neg monocyte: an immunosuppressive phenotype that restrains responses to cancer immunotherapy," Frontiers in Immunology, vol. 10, p. 1147, 2019. 
[6] F. Veglia, M. Perego, and D. Gabrilovich, "Myeloid-derived suppressor cells coming of age," Nature Immunology, vol. 19, no. 2, pp. 108-119, 2018.

[7] M. Lv, K. Wang, and X. J. Huang, "Myeloid-derived suppressor cells in hematological malignancies: friends or foes," Journal of Hematology \& Oncology, vol. 12, no. 1, p. 105, 2019.

[8] A. R. Pyzer, D. Stroopinsky, H. Rajabi et al., "MUC1-mediated induction of myeloid-derived suppressor cells in patients with acute myeloid leukemia," Blood, vol. 129, no. 13, pp. 17911801, 2017.

[9] L. Wang, B. Jia, D. F. Claxton et al., "VISTA is highly expressed on MDSCs and mediates an inhibition of $\mathrm{T}$ cell response in patients with AML," Oncoimmunology, vol. 7, no. 9, article e1469594, 2018.

[10] M. Jun and Chinese Society of Hematology, Chinese Medical Association \& Chinese Society of Hematologist, Chinese Medical Doctor Associations, "Chinese guidelines for diagnosis and treatment of acute promyelocytic leukemia (2014)," Zhonghua Xue Ye Xue Za Zhi, vol. 35, no. 5, pp. 475-477, 2014.

[11] Leukemia, Lymphoma Group CSoHCMA, "Chinese guidelines for diagnosis and treatment of adult acute myeloid leukemia (not APL) (2017)," Zhonghua Xue Ye Xue Za Zhi, vol. 38, no. 3, pp. 177-182, 2017.

[12] V. Bronte, D. B. Chappell, E. Apolloni et al., "Unopposed production of granulocyte-macrophage colony-stimulating factor by tumors inhibits $\mathrm{CD} 8^{+} \mathrm{T}$ cell responses by dysregulating antigen-presenting cell maturation," Journal of Immunology, vol. 162, no. 10, pp. 5728-5737, 1999.

[13] D. Gabrilovich, T. Ishida, T. Oyama et al., "Vascular endothelial growth factor inhibits the development of dendritic cells and dramatically affects the differentiation of multiple hematopoietic lineages in vivo," Blood, vol. 92, no. 11, pp. 41504166, 1998.

[14] A. R. Pyzer, L. Cole, J. Rosenblatt, and D. E. Avigan, "Myeloidderived suppressor cells as effectors of immune suppression in cancer," International Journal of Cancer, vol. 139, no. 9, pp. 1915-1926, 2016.

[15] V. Umansky, C. Blattner, V. Fleming et al., "Myeloid-derived suppressor cells and tumor escape from immune surveillance," Seminars in Immunopathology, vol. 39, no. 3, pp. 295-305, 2017.

[16] J. Zhou, S. S. Donatelli, D. L. Gilvary et al., "Therapeutic targeting of myeloid-derived suppressor cells involves a novel mechanism mediated by clusterin," Scientific Reports, vol. 6, no. 1, p. 29521, 2016.

[17] D. I. Gabrilovich and S. Nagaraj, "Myeloid-derived suppressor cells as regulators of the immune system," Nature Reviews Immunology, vol. 9, no. 3, pp. 162-174, 2009.

[18] A. M. K. Law, F. Valdes-Mora, and D. Gallego-Ortega, "Myeloid-derived suppressor cells as a therapeutic target for cancer," Cells, vol. 9, no. 3, p. 561, 2020.

[19] K. Chikamatsu, K. Sakakura, M. Toyoda, K. Takahashi, T. Yamamoto, and K. Masuyama, "Immunosuppressive activity of CD14+ HLA-DR - cells in squamous cell carcinoma of the head and neck," Cancer Science, vol. 103, no. 6, pp. 976983, 2012.

[20] A. Huang, B. Zhang, B. Wang, F. Zhang, K. X. Fan, and Y. J. Guo, "Increased CD14+HLA-DR-/low myeloid-derived suppressor cells correlate with extrathoracic metastasis and poor response to chemotherapy in non-small cell lung cancer patients," Cancer immunology, immunotherapy : CII, vol. 62, no. 9, pp. 1439-1451, 2013.
[21] X. K. Yuan, X. K. Zhao, Y. C. Xia, X. Zhu, and P. Xiao, "Increased circulating immunosuppressive CD14+HLA-DR-/low cells correlate with clinical cancer stage and pathological grade in patients with bladder carcinoma," Journal of International Medical Research, vol. 39, no. 4, pp. 1381-1391, 2011.

[22] K. De Veirman, E. Van Valckenborgh, Q. Lahmar et al., "Myeloid-derived suppressor cells as therapeutic target in hematological malignancies," Frontiers in Oncology, vol. 4, p. 349, 2014.

[23] Z. Wang, L. Zhang, H. Wang et al., "Tumor-induced CD14 ${ }^{+-}$ HLA-DR ${ }^{-/ l o w}$ myeloid-derived suppressor cells correlate with tumor progression and outcome of therapy in multiple myeloma patients," Cancer Immunology, Immunotherapy, vol. 64, no. 3, pp. 389-399, 2015.

[24] Q. Tao, Y. Pan, Y. Wang et al., "Regulatory T cells-derived IL35 promotes the growth of adult acute myeloid leukemia blasts," International Journal of Cancer, vol. 137, no. 10, pp. 2384-2393, 2015.

[25] J. Wang, Q. Tao, H. Wang et al., "Elevated IL-35 in bone marrow of the patients with acute myeloid leukemia," Human Immunology, vol. 76, no. 9, pp. 681-686, 2015.

[26] H. Huang, G. Zhang, G. Li, H. Ma, and X. Zhang, "Circulating CD $14^{+}$HLA-DR ${ }^{-/ l o w}$ myeloid-derived suppressor cell is an indicator of poor prognosis in patients with ESCC," Tumour Biology, vol. 36, no. 10, pp. 7987-7996, 2015.

[27] C. Wu, X. Wu, X. Zhang et al., "Prognostic significance of peripheral monocytic myeloid-derived suppressor cells and monocytes in patients newly diagnosed with diffuse large b-cell lymphoma," International Journal of Clinical and Experimental Medicine, vol. 8, no. 9, pp. 15173-15181, 2015. 